\title{
At the 'heart' of the COVID-19 outbreak: early cardiac implications and mitigating strategies
}

Robin $\underline{\text { Cherian }}^{1,2}$, MBBS, MRCP, Kian Keong $\underline{P o h^{1,2}}$, MBBChir, FACC

\section{INTRODUCTION}

Coronavirus disease 2019 (COVID-19) has spread across 199 countries, with more than 32,000 deaths reported worldwide and community transmission detected in several countries (including Singapore), as of 29 March 2020. Along with additional healthcare burden, this outbreak has significantly affected delivery of services across institutions and departments. We discuss the implications of COVID-19 on cardiology practice, as well as the strategies for mitigating risks and ensuring continued delivery of services.

COVID-19, with its similarity to severe acute respiratory syndrome (SARS), evokes painful memories among the cardiology fraternity in Singapore. In 2003, one of the first cases of SARS in Singapore was a female patient who was admitted to the cardiology ward with a diagnosis of 'decompensated heart failure'. She deteriorated in the ward and required resuscitation and intubation in the cardiac intensive care unit. A cardiologist, five junior residents and six nurses were inadvertently exposed to the disease and contracted SARS. The cardiac intensive care unit had to be shut down. One young resident eventually succumbed to the disease. While this case clearly illustrates the risks that healthcare workers are exposed to during a deadly outbreak, it also demonstrates the perils of misdiagnosing a respiratory condition as a cardiac illness and the vulnerability of clinical services in the face of an infectious epidemic.

\section{CARDIAC IMPLICATIONS}

COVID-19 is primarily an acute respiratory infection that presents with a wide range of acuity.${ }^{(1)}$ Early cardiac implications include complications directly linked to the disease process, outcomes modified by underlying cardiac comorbidities and cardiac conditions exacerbated by viral illness. Diagnosis can be especially challenging because of the atypical presentations of the disease with overlapping clinical symptomatology and radiology findings. Of particular concern is staff and patient safety due to the risk of infection from inadvertent exposure and nosocomial spread.

SARS and Middle East respiratory syndrome (MERS) were linked to acute myocarditis, acute myocardial infarction and rapid-onset heart failure. ${ }^{(2,3)}$ While the case fatality rate of COVID-19 appears much lower than those of SARS and MERS, early case reports of COVID-19 patients show that cardiac complications are common. In a case report of 138 hospitalised COVID-19 patients, $26 \%$ required admission to the intensive care unit (ICU), of which a significant proportion (44\%) were due to cardiac arrhythmia. Acute cardiac injury was seen in $7.2 \%$ of the entire cohort of patients, cardiac arrhythmia in $16.7 \%$ and shock in $8.7 \%{ }^{(4)}$ In another study of 52 patients with COVID-19 admitted to the ICU for severe illness, cardiac injury was seen in $23 \%$ of patients, with non-survivors showing a significantly higher incidence (28\% vs. $15 \%)$. ${ }^{(5)}$ Evidence of cardiac injury in COVID-19 patients was also demonstrated in other studies, including that of Chen et al, which revealed elevated cardiac enzymes in $13 \%$ of patients who were admitted to the hospital. ${ }^{(6)}$ In another series, Huang et al showed that $31 \%$ of patients who required ICU care had elevated troponins compared to $4 \%$ of those who did not. ${ }^{(7)}$ Whether this points to myocardial injury from acute illness or myocarditis caused by COVID-19 needs to be further studied.

Chronic medical illnesses are common in COVID-19 patients who are admitted to hospital, with one study showing $40 \%$ with underlying cardiovascular and cerebrovascular diseases. ${ }^{(6)}$ However, in the aforementioned study on ICU patients, only $10 \%$ of patients were found to have prior cardiac illness with no difference between survivors and non-survivors. Incident cerebrovascular disease, however, was significantly different between survivors $(0 \%)$ and non-survivors $(13 \%) .{ }^{(5)}$ Huang et al showed that the incidence of cardiovascular disease in patients who were admitted to ICU was higher compared to those who were not admitted to the ICU (23\% vs. $11 \%)$. ${ }^{(7)}$ We will have greater clarity on this when more epidemiological data is published.

It is known that viral illnesses can exacerbate cardiac conditions. ${ }^{(8)}$ Acute exacerbations are common in patients with heart failure as well as in those with coronary artery disease. In patients with poor cardiac reserve, increased demand imposed by viral illnesses is poorly tolerated. Systemic inflammatory responses to viral illnesses are known to destabilise plaques. Similarly, it is expected that patients with COVID-19 infections, especially those with mild disease, may primarily present with a cardiac illness.

Shortness of breath was a presenting symptom in $31 \%$ of patients and chest pain in $2 \%$ in one study. ${ }^{(6)}$ Additionally, fever was not present on admission in $17 \%$ and bilateral chest infiltrates were seen in $75 \%$ of patients. These features may be mistaken for a cardiac presentation, as acute pulmonary oedema can have similar features. These atypical presentations and potential for misdiagnosis have serious implications for staff and patient safety, as inadvertent exposure may lead to infections in staff as well

${ }^{1}$ Department of Cardiology, National University Heart Centre Singapore, ${ }^{2}$ Yong Loo Lin School of Medicine, National University of Singapore, Singapore Correspondence: Dr Robin Cherian, Consultant, Department of Cardiology, National University Heart Centre Singapore, NUHS Tower Block, Level 9, 1E Kent Ridge Road, Singapore 119228. robin_cherian@nuhs.edu.sg 
as nosocomial outbreaks - the effects of which can significantly compromise health systems and services. Particular care needs to be taken to prevent such events. This is even more important in patients who require aerosolising procedures such as non-invasive ventilation; such procedures, when performed inadvertently on a COVID-19 patient, can lead to super spreader nosocomial events, as was the case during SARS. ${ }^{(9,10)}$ This will affect large numbers of staff and patients through airborne transmission. Time-sensitive cardiac emergencies, such as in primary percutaneous coronary intervention for acute ST-elevation myocardial infarction, present additional challenges due to the limited opportunity for risk stratification prior to intervention, thereby increasing the risk of staff exposure. Not uncommonly, respiratory and cardiac conditions, such as heart failure and pneumonia from COVID-19, may co-exist.

Additional challenges exist with regard to service delivery during an outbreak. It is common to segregate clinical areas into 'clean' and 'dirty' areas to limit the effects of inadvertent exposure and to prevent the spread of infection. This can be particularly challenging for cardiology units due to the wide range of services that is spread over various clinical areas of the hospital, as well as the significant staff and patient movement between the 'clean' and 'dirty' areas. Cross coverage also becomes challenging due to the degree of subspecialisation in services.

Furthermore, training and research activities, which are of great importance to an academic centre, are often curtailed during such an outbreak. Large group training sessions are reduced for both undergraduate and postgraduate education. Medical students are not posted to cardiology wards during this outbreak. Small group teaching sessions with standardised patients (i.e. well volunteers) are encouraged instead. Conventional cardiology live meetings are replaced with continual medical education events via teleconference. Patient participation in research is also discouraged due to the risk of infections, while all recruitments for clinical trials are halted. In the long run, this will severely affect research output.

\section{RISK MITIGATION AND ENSURING SERVICE DELIVERY}

The various challenges faced by cardiology practice invite the need for risk mitigating strategies to promote staff and patient safety, and to maintain delivery of essential services. Risk mitigating strategies include early screening and risk stratification, clear workflows, personal protection and staff segregation. High index of suspicion and enhanced surveillance are required to identify cases early (especially in those who present atypically) and for right siting. Patients with underlying cardiovascular conditions may need to be prioritised for treatment due to the higher risk of adverse outcomes. Particular care has to be taken in patients who require critical care interventions and aerosolising procedures, as misdiagnosis can have serious implications. In addition, strict adherence to guidelines for personal protection equipment and hand washing is required to prevent staff exposure and nosocomial spread. Staff segregation also ensures redundancy in case of sickness or quarantine.

During an outbreak, technology can be embraced for continued delivery of services. Patients who require essential services should not be denied what is clinically indicated. Vaccination needs to be current and guideline-directed treatment of cardiac illnesses should also be reinforced to ensure that cardiac conditions remain stable. Potential methods to sustain services while reducing the risk of infection include tele-monitoring and video consultation, while online videoconferencing platforms can be used for continuous medical education activities.

\section{CONCLUSION}

The SARS outbreak caught Singapore unawares, and we lost a few of our own in that battle. The level of preparedness since then has been considerable. ${ }^{(11,12)}$ While COVID-19 poses various challenges to cardiology practice and service delivery, with careful planning, use of technology and adherence to established infection control strategies, we hope to soon overcome this pandemic.

\section{REFERENCES}

1. Paules $\mathrm{Cl}$, Marston HD, Fauci AS. Coronavirus infections-more than just the common cold. JAMA 2020 Jan 23. https://doi.org/10.1001/jama.2020.0757. [Epub ahead of print]

2. Alhogbani T. Acute myocarditis associated with novel Middle East respiratory syndrome coronavirus. Ann Saudi Med 2016; 36:78-80.

3. Peiris JS, Chu CM, Cheng VC, et al. Clinical progression and viral load in a community outbreak of coronavirus-associated SARS pneumonia: a prospective study. Lancet 2003; 361:1767-72.

4. Wang D, Hu B, Hu C, et al. Clinical characteristics of 138 hospitalized patients with 2019 novel coronavirus-infected pneumonia in Wuhan, China. JAMA 2020; 323:1061-9.

5. Yang $X, Y u Y, X u$ J, et al. Clinical course and outcomes of critically ill patients with SARS-CoV-2 pneumonia in Wuhan, China: a single-centered, retrospective, observational study. Lancet Respir Med 2020; 8:475-81.

6. Chen N, Zhou M, Dong X, et al. Epidemiological and clinical characteristics of 99 cases of 2019 novel coronavirus pneumonia in Wuhan, China: a descriptive study. Lancet 2020; 395:507-13.

7. Huang C, Wang Y, Li X, et al. Clinical features of patients infected with 2019 novel coronavirus in Wuhan, China. Lancet 2020; 395:497-506.

8. Kytömaa S, Hegde S, Claggett B, et al. Association of influenza-like illness activity with hospitalizations for heart failure: the Atherosclerosis Risk in Communities Study. JAMA Cardiol 2019; 4:363-9.

9. Fowler RA, Guest CB, Lapinsky SE, et al. Transmission of severe acute respiratory syndrome during intubation and mechanical ventilation. Am J Respir Crit Care Med 2004; 169:1198-202

10. Yu IT, Xie ZH, Tsoi KK, et al. Why did outbreaks of severe acute respiratory syndrome occur in some hospital wards but not in others? Clin Infect Dis 2007; 44:1017-25

11. Lum LHW, Tambyah PA. Outbreak of COVID-19 - an urgent need for good science to silence our fears? Singapore Med J 2020; 61:55-7.

12. Hsu LY, Chia PY, Vasoo S. A midpoint perspective on the COVID-19 pandemic. Singapore Med J 2020; 61:381-3. 